\title{
Slow flows of yield stress fluids: yielding liquids or flowing solids?
}

\author{
P. Coussot \\ Université Paris-Est, Laboratoire Navier (ENPC-IFSTTAR-CNRS), Champs sur Marne, France
}

\begin{abstract}
Yield stress fluids (YSF) exhibit strongly non-linear rheological characteristics. As a consequence they develop original flow features (as compared to simple fluids) under various boundary conditions. This paper reviews and analyze the characteristics of a series of slow flows (just beyond yielding) under more or less complex conditions (simple shear flow, flow through a cavity, dip-coating, blade-coating, Rayleigh-Taylor instability, Saffman-Taylor instability) and highlight some of their common original characteristics: i) a transition from a solid regime to a flowing regime which does not correspond to a true "liquid state", the flow in this regime may rather be seen as a succession of solid states during very large deformation; ii) a strong tendency to localization of the yielded regions in some small region of the material while the rest of the material undergoes some deformation in its solid state; iii) the deformation of YSF interface with another fluid, in the form of fingers tending to penetrate the material via a local liquefaction process. Finally these observations suggest that for slow flows of YSF are a kind of extension of plastic flows for very large deformations and without irreversible changes of the structure. This suggests that the field of plasticity and the field of slow flows of YSF could benefit from each other.
\end{abstract}

\section{Introduction}

Although certainly not realized by the man of the street, yield stress fluids (YSF) such as foams, emulsions, paints, colloids, physical gels, etc, play a major role in our everyday life, and arouse a growing interest in rheology [Coussot 2017]. These materials behave as solids under a critical stress and yield, in the sense that flow in an (apparent) liquid regime, when the critical (yield) stress is overcome. The main fields of application, use, and research, are oil industry and construction, both fields in which the specific yield stress behavior of the materials (cement paste, drilling fluids, foams, fresh concrete) is well recognized and taken into account. In other fields such as cosmetics, foodstuffs, treatments of mining residues, slewage sludges, ceramics, natural flows, etc., the yielding behavior and some related parameters, are often used to characterize the material and adjust the formulation. The flow characteristics of such material type under specific boundary and initial conditions are less often studied in these fields. Yet YSF flows exhibit original characteristics which can have a strong impact on the process efficiency.

In fluid mechanics the beautiful and scientifically attractive features of flows under specific boundary conditions has drawn for a long time the attention of people. This led to various websites, videos and books, an enthusiasm illustrated by the competition organized each year by the Division of Fluid Dynamics of the American Physical Society. With a similar motivation, in the field of non-Newtonian fluid mechanics, D.V. Boger and K. Walters published a book essentially focused on flows of viscoelastic liquids [Boger and Walters 1993] and the (American) Society of Rheology started to organize in 2017 a "Gallery of Rheology" contest. The starting point of the present paper was a modest contribution in a similar spirit concerning yield stress fluid flows. We review a series of yield stress fluid flows under complex boundary conditions and for which we have a detailed information on the internal flow characteristics. In order to clearly distinguish this field from that of simple fluids 
we focus on slow flows, that we define as flow conditions for which the yielding characteristics of the fluid are dominant, i.e. the (maximum) stress needed to maintain the flow is close to the critical stress needed to induce a flow in an apparent liquid regime. A more quantitative definition will be given in the paper.

More technical and complete reviews on different aspects of yield stress fluid behavior or flow characteristics may be found in recent publications such as [Coussot 2014, Balmforth et al. 2014, Bonn et al. 2017, Rheologica Acta Special Issue on yield stress fluids 2017]. Here we will rather focus on the original flow features of yield stress fluids under various more or less complex conditions: flow in a rheometer geometry, flow through a cavity larger than the main conduit, displacement of a long object through a bath of YSF, blade-coating (a process widely used in civil engineering for example for paints, mortars, etc), traction of a YSF between two solid surfaces or as a result of gravity, destabilization of the interface with another fluid (Saffman-Taylor instability, Rayleigh-Taylor instability). For each of them, we will consider the distinctive characteristics of the flow with regards to what would be obtained with a simple fluid and, as far as possible, we will attempt to explain the physical origin of these differences with simple arguments.

During these review and analysis some original characteristics of YSF flows will be highlighted, which will structure the plan of the paper: i) a transition from a solid regime to a flowing regime which does not correspond to a true "liquid state", the flow in this regime may rather be seen as a succession of solid states during very large deformation (Section 2); ii) a strong tendency to localization of the yielded regions in some small region of the material while the rest of the material undergoes some deformation in its solid state (Section 3); iii) the deformation of YSF interface with another fluid, in the form of fingers tending to penetrate the material via a local liquefaction process (Section 4). Finally these observations show that slow flows of YSF are a kind of extension of plastic flows for very large deformations and without irreversible changes of the structure.

\section{The different rheological and physical states of yield stress fluids}

\section{Solid regime}

The rheological behavior of a yield stress fluid, apparently intermediate between a solid and a fluid, is something unexpected from the usual or more sophisticated [Coleman et al. 1966] approaches of material behavior in continuum mechanics, i.e. which distinguished two main classes of materials, solids and fluids. This is also an original state of matter from the physical point of view [Coussot 2017]. This leads to original flow properties even under homogeneous stress conditions. Indeed let us consider a series of creep tests with a given yield stress material, consisting to apply a given stress to a sample and follow the deformation in time, then start again the same test at another stress level on the material placed in the same initial state. For a stress below a critical value we get a response typical of a solid [Cottrell 1964] (see Figure 1): the deformation first increases, then very rapidly tends to saturate and reach a plateau [Coussot et al 2006]. Remarkably, for some YSF, if we maintain the stress application for a long time, we may observe an extremely slow "flow", in fact a very slight increase of the apparent deformation [Lidon et al. 2017]. This can nevertheless not be considered as reflecting a standard viscous behavior associated with the flow of a given material, since in that case the apparent shear rate continuously decreases in time (see e.g. pink curve in Figure 1), and the deformation remains very small. This more likely reflects some slight rearrangements of the structure and/or localization of the deformation. For solids or glasses a similar effect may be observed, which is called aging. This effect, which occurs only for some materials, and does not induce significant 
deformation over usual times of observation, will be left apart in the following discussion, and we will essentially be concerned by the approximate deformation plateau.

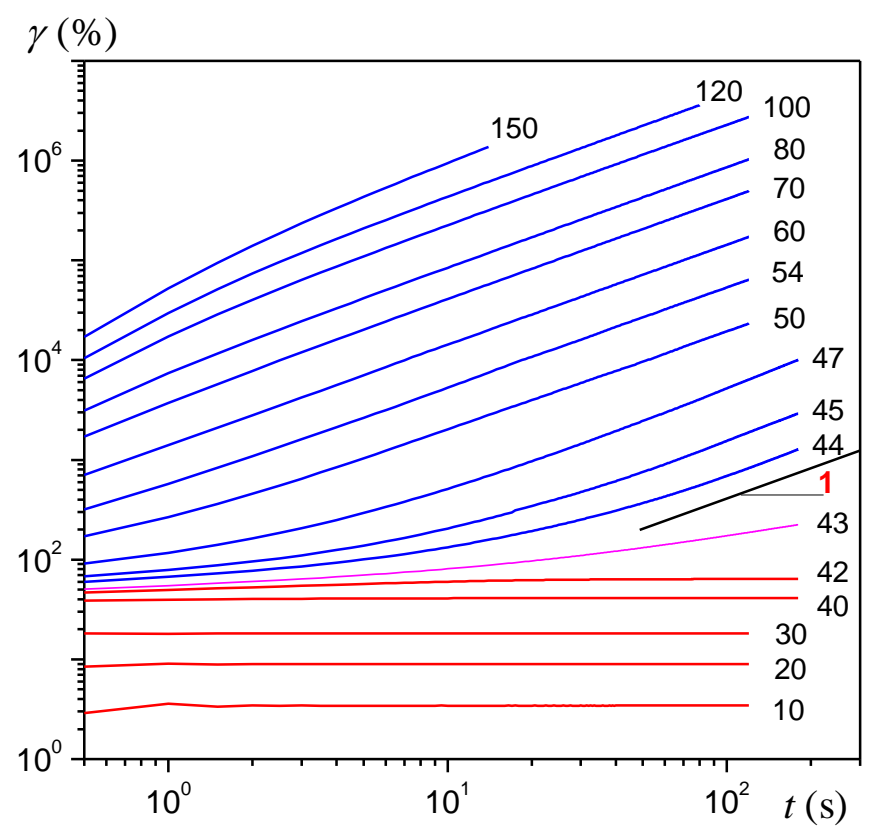

Figure 1: Deformation vs time for creep tests at different stress values (the numbers in front of each curve correspond to the stress values in Pascals) for a direct concentrated emulsion (82\%). The liquid regime corresponds to curves tending to a slope 1 (blue curves), the solid regime corresponds to curves tending to a slope 0 (red curves). For the pink line we have at a given time $(t): \ln (\gamma)=n \ln (t)$ with $n<1$, which implies $\dot{\gamma}=n t^{n-1}$ and thus $\dot{\gamma} \rightarrow 0$ when $t \rightarrow \infty$.

In order to further appreciate the behavior of the material in this solid regime it is useful to plot the stress vs the level of the deformation plateau, as is common for a solid material. In that case, in some range of (small) deformations, the slope of the corresponding curve is constant (see Figure 2a). Moreover, in this range, if we suddenly release the stress, the sample goes back to its initial position (deformation equal to zero). Thus we are in a linear elastic regime. Then, for larger stresses, the slope of the stress vs deformation curve decreases progressively. In this regime, when the stress is released, the deformation does not go back to zero, but to a finite value, which increases with the previously applied stress. This means that the material is elastoplastic, it undergoes a part of irreversible deformations. In the example presented in Figure $2 a$ it is remarkable that in this regime the elastic component of the behavior still remains linear with the same slope as in the purely elastic regime. Note that here we only considered final states of the material under different stress values, but since the transformations are not instantaneous, some viscous component of the behavior might also be considered.

Such a behavior is naturally associated with a jammed structure of the material [Liu and Nagel 1998]. What does mean jamming here? This is the fact that the components of the material are elements of a structure spanning throughout the sample, and in which neighboring elements interact significantly even at rest, so that thermal agitation alone cannot break the structure. Under such conditions some force applied to the system will induce a deformation of the structure, associated with slight relative displacements of neighboring elements. For sufficiently small stress, the structure will be fully 
recovered when releasing the stress. For larger stress (but still below the critical one) there might be locally some breakage of the link between two (initially) neighboring elements, which will not be recovered after stress release. These local link breakage are also named "plastic events" in physics of jammed systems, and might be at the origin of plasticity in the solid regime of yield stress fluids [Hébraud et al. 1997].
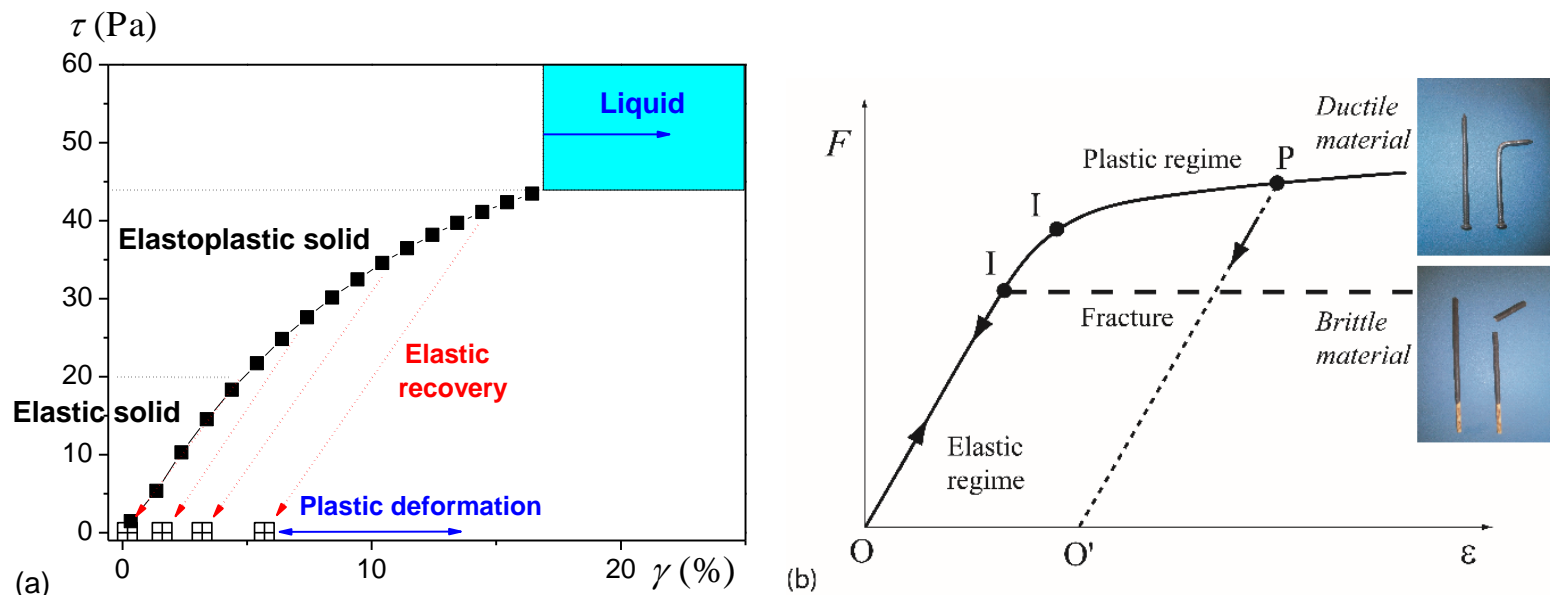

Figure 2: Stress vs final deformation from creep tests for (a) a typical YSF (here an emulsion, data from Maimouni et al 2016) and (b) typical solids (brittle or ductile).

\section{Liquid regime}

The above scheme is conceptually analogous to that developed for a solid [see for example Cottrell 1964, Tabor 1991]. For example, the local link breakages correspond to dislocations for pure solids. For such a standard solid the next step, i.e. for a sufficiently large stress, is breakage or plastic flow and localization, depending on whether we are dealing with a brittle or ductile material respectively (see Figure 1b). A fundamental characteristics of this behavior for solids is that the transformation of the material is irreversible beyond some point (I in Figure $2 \mathrm{~b}$ ): in general it will not be possible to get back the material with exactly the same rheological behavior, because the structure has been modified and cannot recover its initial state by itself. That in particular means that, even if in some cases we can observe some (plastic) flow the apparent behavior of the material continuously evolves, and this corresponds to an irreversible alteration of the material.

The situation is fundamentally different for what we call a yield stress fluid: for a stress larger than a critical value, i.e. the yield stress $\left(\tau_{c}\right)$, the deformation does not reach a plateau, it continuously increases and its rate of increase finally reaches a steady value. Thus we can consider that we have a steady state flow at a constant shear rate. This is what we will call here the "liquid regime". The remarkable point, as compared to standard solids, is that despite the very large deformations possibly undergone by the material in this regime, the material transformations are reversible: if we release the stress, and prepare the material in the same way as previously, we will get the same evolution of the deformation in the solid and liquid regime, and finally the same apparent viscosity in steady state flow. That means that (i) the structure can support thermal agitation without breaking, (ii) it may be fully broken so as to get a flow, and (iii) it fully recovers its initial characteristics after full disintegration of the structure. Note that, since the structure is not fully broken in the solid regime (despite some possible local plastic events), we can presume that in the solid regime a slight 
heterogeneity of the stress distribution will not have significant impact and we will get a roughly homogeneous macroscopic deformation of the sample. On the contrary, in the liquid regime, since the structure is broken we can anticipate that some slight heterogeneity can easily lead to some significant heterogeneity of the deformation field (see below).

How can we explain such differences between a plastic solid and a YSF? This is likely due to the nature of the elements and their mutual interactions, and two essential ingredients might be necessary: (a) the elements develop "soft" elastic interactions, in the sense that their mutual forces vary relatively slowly with the distance between elements, so that local relaxation is fast; (b) the elements are in a disordered configuration, i.e. there is no particular ordered structure taking place over a large number of elements, and this disorder is such that two such configurations cannot be distinguished. With such characteristics we start to understand the YSF properties: below a critical stress it is possible to somewhat deform the material without breaking the structure; beyond this critical stress the structure is broken but the new (still disordered) structure obtained after this breakage does not significantly differ from the initial one, and eventually at rest the material rapidly relaxes towards its initial structure associated to the solid state. Finally we are faced with a unique kind of material: a self-healing solid.

The situation that we here called "liquid regime" differs from the standard definition of a liquid in physics, i.e. associated with densely packed molecules undergoing thermal agitation. Here the effects of thermal agitation remain negligible as compared to the jamming or viscous effects, so that by no way the structure resembles that of a liquid as above described. And since as soon as the stress is released, the flow stops and the material recovers an apparent solid state, we can finally consider that the liquid regime just corresponds to a large, continuously increasing, deformation, of a selfhealing solid material. Such materials are the object of physical studies which consider an extension of the concept of plastic events to the liquid regime [Sollich et al (1997), Maloney and Lemaitre (2006)].

Referring to the standard picture of a plastic material slowly flowing under a constant critical stress, it follows that the behavior of a YSF under slow flow conditions would express as $\dot{\gamma} \neq 0 \Rightarrow \tau=\tau_{c}$. In fact, such an expression cannot be considered as a proper constitutive equation since it does not allow to define the flow rate, and measurements fortunately show that the stress increases with the flow rate (see Figure 1). It is now well recognized that under simple shear the flow curve (i.e. shear stress vs shear rate in steady state) of simple YSF (i.e. exhibiting negligible thixotropic characteristics) is well represented, over several decades of shear rates, by a Herschel-Bulkley model, which expresses as:

$\dot{\gamma} \neq 0 \Rightarrow \tau=\tau_{c}+k \dot{\gamma}^{n}$

in which $\tau$ is the shear stress amplitude, $\dot{\gamma}$ the shear rate amplitude, and $k$ and $n$ are material parameters (generally $n$ is situated between 0.3 and 0.5 ). Such an expression is the point at which we start to depart from the pure plastic behavior and we have to consider that viscous effects take place in the liquid regime. This increase of the stress with the flow rate means that the structure does not have enough time to fully relax so as to reach exactly the same state as at rest, between two steps of deformation, so that the deformation at the next step requires a somewhat larger stress than that needed for the structure initially at rest.

\section{Simple shear flows}




\section{Slow flows}

We can now define more precisely what we call "slow flows", on which we focus in this paper. They correspond to situations for which the yielding characteristics play a major role. For a simple shear this is obtained when the first (constant) term of the stress expression (1) is much larger than the second term. This corresponds to $\tau_{c} / k \dot{\gamma}^{n}>>1$. On the contrary, if $\tau_{c} / k \dot{\gamma}^{n}<<1$ the material essentially behaves as a power-law fluid with flow characteristics approaching those of simple liquids. For 3D flow the situation is a priori more complex. The standard description (but not fully validated yet) of the constitutive equation in $3 \mathrm{D}$ relies on an extrapolation of the simple shear behavior assuming that the stress tensor depends essentially on the second invariant $\left(D_{I I}\right)$ of the strain rate tensor (D) [Oldroyd 1947]. It assumes that the stress tensor $(\boldsymbol{\Sigma})$ is a sum of one term related (and likely proportional) to the yield stress in simple shear, and one related to the additional stress term related (with a factor proportional to $k$ ) to the second term of (1), which gives $\boldsymbol{\Sigma}=-p \mathbf{I}+\tau_{c} \mathbf{D} / \sqrt{-D_{I I}}+\left(2^{n} k /\left(-D_{I I}\right)^{(1-n) / 2}\right) \mathbf{D}$, where $p$ is the pressure and $\mathbf{I}$ the unit tensor. Then we can compare the invariants of the different components of this stress tensor expression (leaving apart the pressure term): the invariant of the second term is $\tau_{c}$, the invariant of the third term is $k\left(2 \sqrt{-D_{I I}}\right)^{n}$, which we can roughly estimate as $k(V / l)^{n}$, in $V$ is a characteristic flow velocity and $l$ a characteristic flow length. Finally a generalization of our criterion of slow flow is the following approximate criterion:

$$
B i=\frac{\tau_{c}}{k(V / l)^{n}}>1
$$

in which $B i$ is the Bingham number.

\section{Localization in simple shear rheometrical tests}

It remains that the stress increase at low shear rate is rather slow, which particularly appears when the flow curve is represented in a logarithmic scale: the stress tends to a plateau associated with the yield stress when $\dot{\gamma} \rightarrow 0$. A consequence of this behavior is that the exact determination of the behavior (in terms of shear rate associated with a given value of shear stress) is difficult as it requires to impose a perfectly homogeneous stress throughout the sample, otherwise the effective shear rate can differ from the apparent one. As a corollary, a localization of the deformation is highly probable at the approach of the yield stress. To illustrate that point let us consider the flow in a cone and plate geometry with a very small angle. The applied torque $M$ is transmitted through conical surfaces with decreasing angles towards the lower plate, so that the shear stress in each of these surfaces writes $\tau=3 M / 2 \pi R^{3}$. Now, if the curvature of the periphery of the sample is not ideal, i.e. not spherical, $R$ varies from one layer to another. This is a typical phenomenon observed with cone and plate or parallel disk geometry, the peripheral free surface tends to curve inwards, leading to a smaller radius in the middle of the sample. Under these conditions the shear stress may easily vary by several percent or tens of percent over the gap. For example we can frequently observe an inward curvature leading to a smaller radius by about $0.5 \mathrm{~mm}$; for a typical radius of $3 \mathrm{~cm}$ along the walls this leads to a stress at the necking point larger by $5 \%$. For rheological parameters with typical values such that $k / \tau_{c}=3$ and $n=0.5$, we can have an apparent flow for an apparent stress $\tau=\tau_{c}$, whereas the effective stress is $\tau=1.05 \tau_{c}$ in some central region, so that the shear rate varies from 0 along the walls to $0.023 \mathrm{~s}^{-1}$ in the central region. Thus the effective shear rate is strongly heterogeneous in the 
gap, and the apparent shear stress does not provide the exact stress value associated with the mean shear rate. At slightly lower stress value we can even expect a complete localization of the flow in a thin central region, so that the rheometrical measurements just provide an estimation of the yield stress, but are not at all relevant concerning the effective shear rate. This implies that it is rather difficult to get relevant data concerning the effective shear rate value in the sample below some value, of the order of $0.1 \mathrm{~s}^{-1}$ in the present example.

Another difficulty is that, in order to reach steady state we have to impose a flow during a time sufficient for the deformation to overcome the critical one associated with the liquid regime. Otherwise we do not observe steady state flow but a stress value associated with some transient behavior in the solid regime.

To sum up, we can hardly consider that under flow conditions a YSF is in a true liquid state. Instead we may see it as a (self-healing) solid material able to widely deform without losing its basic jammed structure, and increasing the flow rate just slightly drifts the structure state from its basic state associated with rest.

\section{The special case of thixotropic yield stress fluids}

For the sake of simplicity, in all the discussion so far we were considering YSF exhibiting negligible thixotropy effects. Such effects take the form of time evolution of the apparent viscosity in the liquid regime, due to some progressive destructuring. This means that now, in contrast with our assumptions above, the structure can significantly evolve when the material is deformed. Under these conditions, if the structure evolutions are irreversible we are not dealing with a YSF. Such a material belongs to the class of YSF only if its structure evolutions are reversible. This implies that a restructuring process is expected under other flow conditions than those which led to destructuring, such as, typically, some period at rest. Thus, even if we can observe trends with some similarity with those above described, the precise characterization of such materials in the solid and in the liquid regime becomes more difficult than for non-thixotropic YSF, since it is now necessary to take into account the impact of the whole flow history on the current behavior. On the basis of observations and reasonings it was suggested (Coussot et al. 2009, Moller et al. 2009, Coussot and Ovarlez 2010), that this situation is essentially encountered with colloidal suspensions of aggregated particles, in which the particles are somewhat dispersed and poorly interact when the material breaks; whereas simple YSF are obtained when the structure is made by concentrating relatively large deformable elements in a limited volume, such as for foams, physical gels, emulsions, etc., which thus rapidly relax and recover a similar structure after breakage. In the present paper we consider only "simple" YSF (Ovarlez et al. 2013), i.e. with negligible thixotropic effects.

Note that the flow of thixotropic materials are extremely prone to develop shear-banding (see e.g. Ovarlez et al. (2009)). This is so because the destructuring process being significant, under some apparent shear imposed by boundary conditions, it may soon become easier for the material to strongly destructure in some specific region, so that its viscosity drops to rather low value, while remaining in its solid state elsewhere.

Wall slip

A trend which can play an important role in YSF flows is wall slip, a situation in which the bulk moves as a rigid block along a smooth solid surface, the flow being concentrated in an extremely thin layer of material with a structure, and thus a behavior, differing from that of the bulk (Cloitre and 
Bonnecaze 2017). The structure of YSF, generally made of elements suspended in a liquid phase, is particularly well fitted to the development of this effect. Indeed a simple extraction of a very small amount of the interstitial liquid from the bulk, or the natural steric depletion of the density of suspended elements at the approach of the wall, are sufficient to form, along the wall, offering a resistance to shear flow smaller than the yield stress (in that aim one just needs to decrease the displacement velocity at a sufficiently low level). As a consequence the YSF bulk can move along the wall thanks to the shearing of this layer, even if the applied stress is smaller than the yield stress. This induces a critical change of the apparent behavior of the material which, although it behaves as a solid unable to flow below a critical stress with rough surfaces, turns to a apparently simple liquid in the same range of stresses with smooth surfaces. An illustration of this effect is shown in Figure 3: a heap of concentrated emulsion lying on a smooth solid surface can flow (as a rigid block) when this surface is inclined, and without leaving tracks of fluid behind, which would be the hallmark of bulk shearing along the solid. There might instead remain a very thin layer of interstitial liquid, soon evaporating. Note that there are nevertheless small tracks remaining along the lateral edges of the sample, likely due to some adherence of the line of contact (see Zhang et al (2017)).

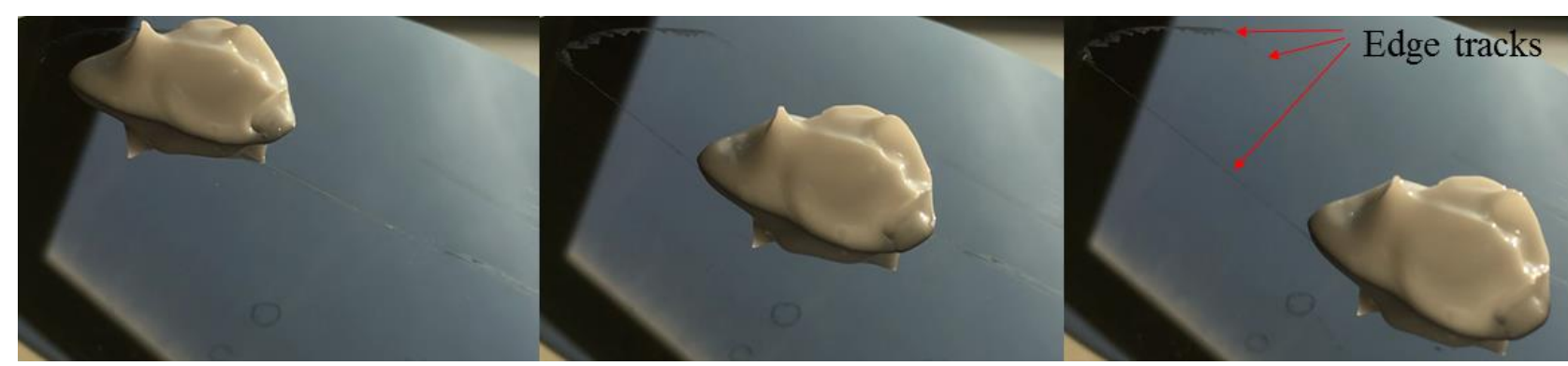

Figure 3: Successive views of a heap of emulsion (yield stress: $40 \mathrm{~Pa}$ ) put on a smooth surface, at times: (a) 0 , (b) $15.6 \mathrm{~s}$, (c) $26 \mathrm{~s}$. The sample length is about $3 \mathrm{~cm}$. [Courtesy Xiao Zhang]

\section{Localization in complex flows}

The original rheological behavior of YSF, including a solid and a liquid regime, obviously leads to particular flow properties. A first trend results from the fact that these two different states depend on the stress value as compared to the yield stress value. For rheometrical flows in simple shear and when the stress field is approximately homogeneous the material will mainly be, as a whole, in a given regime, depending on the applied stress, except at low shear rates due to some residual slight heterogeneity of the stress distribution (see Section 3). As soon as the stress field is significantly inhomogeneous the situation is much more complex: the fluid can flow in its liquid regime in some regions while remaining at rest (while possibly deformed) in other regions; the spatial distribution of these different regions is expected to evolve as the global flow rate is increased, in particular the volume of the liquid region should increase. However the exact distribution of stress, which will determine the distribution of arrested or flowing regions is in general a priori unknown, it results from the interplay between the boundary conditions and the rheological behavior of the material. We start by considering a simple case (flow in a conduit) for which this stress distribution is known and then move to other more complex geometries.

\section{Conduit flow}


The typical situation of a YSF flow through a straight cylindrical conduit (of radius $R$ ) illustrates these characteristics: for slow flows the liquid regime is confined in a thin layer along the wall while the rest of the material in the middle of the conduit advances as a plug; as the flow rate increases the thickness of the sheared layer increases and the plug size decreases. This strongly contrasts with the laminar flow of a Newtonian fluid: in that case the local flow intensity simply increases proportionally to the global flow rate so that the qualitative flow characteristics are independent of the flow rate. In particular, for YSF, the thickness $(e)$ of the sheared layer is expected theoretically to tend to zero when the velocity tends to zero, or equivalently when the Bingham number tends to infinity. More precisely, for a HB fluid, we have $e / R \approx(m+1)^{1 /(m+1)} B i^{-m / 1+m}$ (with $m=1 / n$ and $B i=\tau_{c} R^{n} / k V^{n}$ ) (Chevalier et al (2013)). This poses a serious problem when this thickness falls below the lengthscale of the representative elementary volume of material. In that case the apparent flow properties of the material through the conduit rely on the shearing of a layer of material whose behavior may differ from that of the bulk, so that the macroscopic flow properties may differ from those expected from theory and constitutive equation determined from homogeneous flow conditions. Note that the wall roughness may also play an important role in such a situation, as it can interact with the sheared thickness. It may also lead to a specific situation if it is close to zero (with regards to the size of the elements of the jammed structure), this is wall slip.

\section{Flow through a larger volume}

Let us consider the simple case of a YSF through a straight conduit with a sudden enlargement of the conduit diameter over a limited length before a sudden restriction into the initial diameter. In the main conduit, at large Bingham number, we have essentially a plug flow with a thin sheared layer along the wall. Surprisingly these flow characteristics are weakly modified during the motion through the larger conduit, the entering fluid essentially keeps a plug state, while most of the rest of the fluid in the larger conduit is undeformed (see Figure 4). Finally, only a thin layer of fluid, situated between these two solid regions, is sheared, ensuring the velocity continuity. Moreover the shear rate in this layer is approximately constant. A similar situation, with similar flow characteristics (after rescaling by the plug velocity) and similar thickness of the sheared layer is observed for different values of the Bingham number as long as it remains larger than 1 (Chevalier et al, 2013), but it seems difficult to predict the size of the sheared layer from simple analytical arguments.

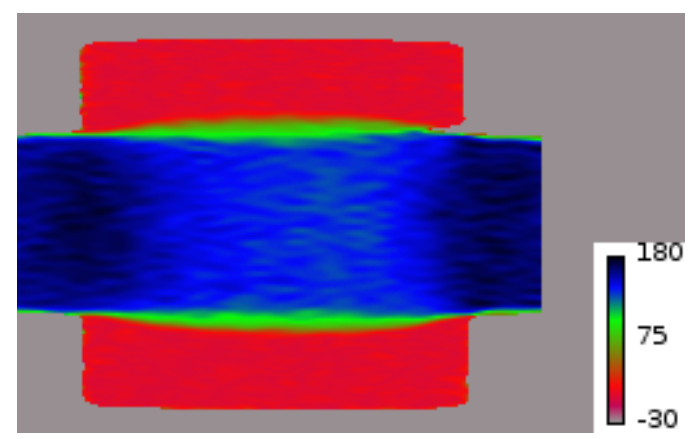

Figure 4: Flow of a yield stress fluid (concentrated emulsion with $\tau_{c}=74 \mathrm{~Pa}$ ) through a sudden enlargement (diameter $7 \mathrm{~cm}$ ) then contraction (back to $3.5 \mathrm{~cm}$ diameter). Velocity map in a longitudinal cross-section for a mean velocity in the small conduit of $0.16 \mathrm{~mm} / \mathrm{s}$. The velocity amplitude (in microns per second) in each voxel is represented 
by a color according to the scale at the bottom right (in microns/s). [Figure 1 of Chevalier et al (2013)].

\section{Displacement of a plate along one of its axis}

A similar result is obtained for a plate moving vertically through a yield stress fluid bath in a container. For a plate penetrating the bath or extracted from the bath (dip-coating) a liquid region exists only in a thin layer along the moving plate, while the rest of the fluid is slightly deformed in its solid regime (see Figure 5). Once again it appears that the shear rate is almost constant in the sheared layer and the size the liquid region does not seem to change when varying the Bingham number in a range above 1, i.e. the flow fields are similar after rescaling by the plate velocity. However, predicting the value of the sheared layer from simple analytical arguments remains challenging.
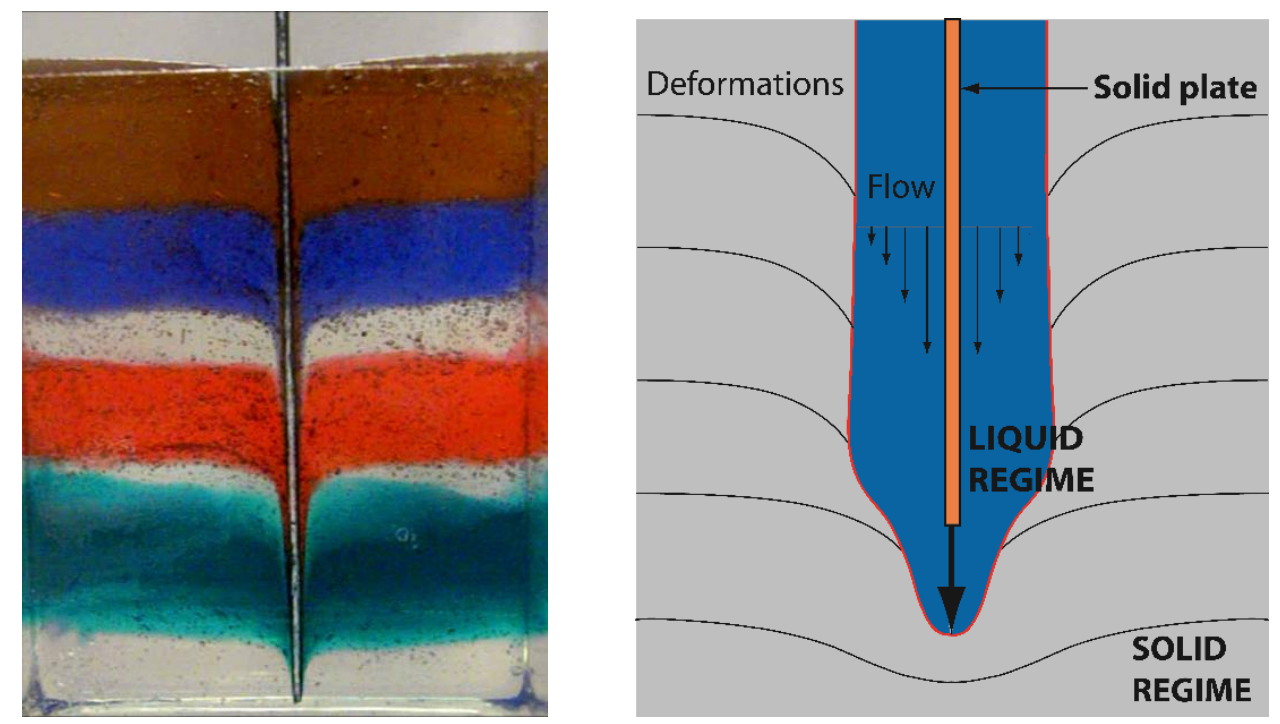

Figure 5: (left) Deformation undergone by a bath of Carbopol gel during the vertical penetration of a plate ( $6 \mathrm{~mm}$ thick) (here seen from its side). The material was initially set up in the form of successive layers with different colors (but same rheological behavior). The deformation of the interfaces, observed along the transparent wall, allows to see the material deformation, and we know from PIV measurements (see Boujlel et al 2012) that this well reflects the deformation inside the bath. Two regions can be distinguished: within a distance of the order of the plate thickness the YSF is strongly deformed; beyond this distance it is slightly deformed. [Courtesy Jalila Boujlel]. (right) Scheme of the deformation field around the plate, as deduced from direct internal measurements of the flow field in time (adapted from Boujlel et al 2012). The solid and liquid regions were identified from the evolution of the total deformation undergone by material elements along their trajectories: the deformation does not overcome a small value in the solid region, while it continuously increases in time and reaches large values in the liquid region. 


\section{Blade coating}

Similar characteristics are observed in blade-coating, although the existing observations are much less precise (Maillard et al (2016)) or obtained from rough numerical simulations (Maillard (2015)). The principle of the experiment is the displacement of a vertical or inclined blade along the surface (and somewhat dipped in) of a uniform YSF layer lying over a horizontal channel. The process leads to the accumulation of an increasing volume of material against the front of the blade, and leaves behind a uniform thickness of material (see Figure 6). The data show that the flow is essentially the result of the displacement of a rigid YSF block attached to the blade, relatively to a material layer at rest along the bed, while some thin layer is sheared to ensure the contact between these two solid regions. Obviously, since the region stuck to the blade is continuously fed by additional material, the above description is an approximation valid over a short displacement. Finally rough observations and numerical simulations tend to show that the sheared layer thickness does not change significantly as the velocity is increased.

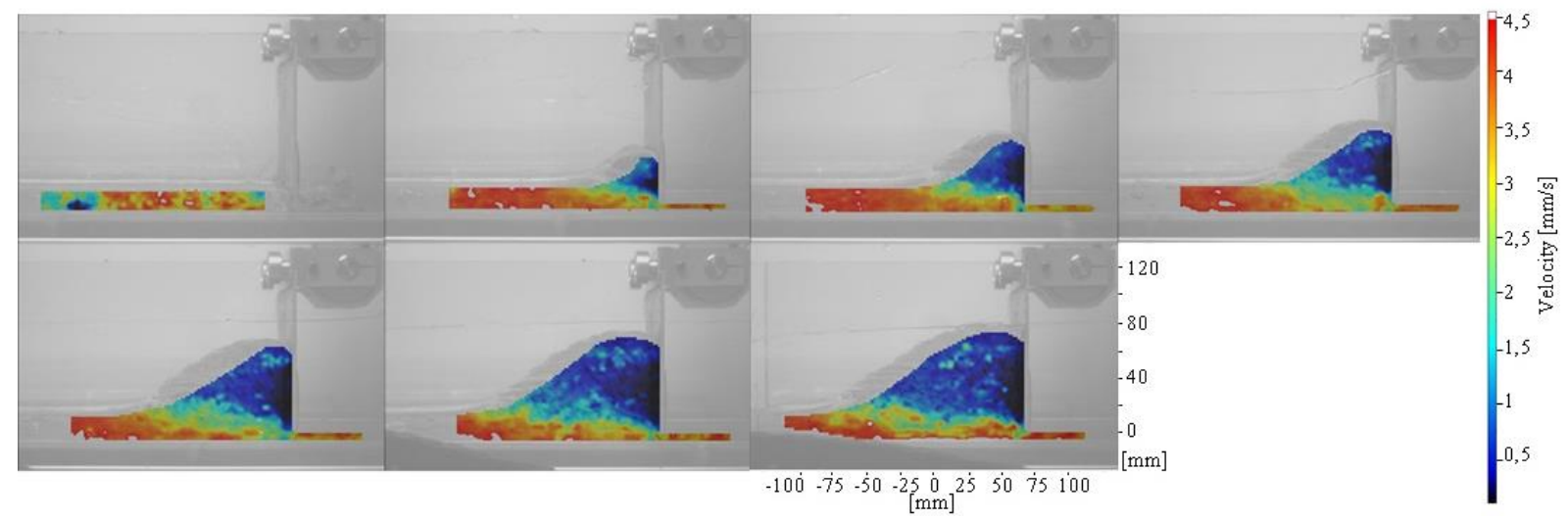

Figure 6: Evolution of the experimental horizontal velocity field during the blade-coating in a channel filled with a yield stress fluid (Carbopol gel, or yield stress $98 \mathrm{~Pa}$. The blade velocity is $\mathrm{V}=5 \mathrm{~mm} / \mathrm{s}$, the initial (uniform) height of fluid is $17 \mathrm{~mm}$, the depth of penetration of the blade into the fluid is $7 \mathrm{mmm}$ ). The pictures correspond to successive steps of displacement of $100 \mathrm{~mm}$. In this representation the blade has a velocity equal to zero (dark blue) while the channel has a velocity V (red).

\section{Synthesis}

From the above description we can deduce several important points:

- Despite complex boundary conditions, YSF tend to develop a deformation field such that the liquid region undergoes essentially a simple shear; this result is absolutely not trivial, for example the entrance flow in a die was considered as involving mainly an elongational flow involving all the material volume (Benbow and Bridgewater 1993).

- The resulting sheared regions exhibit some analogy with shear-bands as observed in plasticity (Nadai 1950): indeed, they support the entire relative motion of the material and the boundaries, their thickness is in general small compared to the sample size, the shear rate is almost constant inside the band and their thickness does not vary significantly with the velocity for slow flows.

- In contrast with usual solid plastic materials in which shear-bands can develop, here the large deformation undergone by the YSF in the band do not alter irreversibly its mechanical behavior. 
Such properties are characteristics of plastic flows, but with the specificity that the YSF is still able to self-heal. This suggests that a specific treatment of slow flows of YSF should be developed, inspired from plastic flows or, conversely, slow flows of YSF provide a way to study plastic flows.

\section{Deformation of YSF interfaces}

Let us now examine what happens for less constrained flows of YSF, i.e. a flow with large interfaces with a simpler fluid (air or a Newtonian liquid).

Localization in (attempted) extensional flows: jets and droplets

Let us consider the formation of a droplet, which is generally obtained by pushing a fluid through an opened conduit. For a simple fluid this forms a jet which will separate in droplets after some distance depending on the viscosity, the surface tension and the flow rate. This phenomenon is governed by the Plateau-Rayleigh instability. The instability basically occurs because the surface energy of a cylinder of fluid is larger than for the same fluid volume separated in spherical droplets of a particular diameter. During the separation, viscous effects can nevertheless slow down the process so that this separation will occur in a characteristic time depending on the balance between capillary and viscous effects. A similar situation is expected for a YSF, but now the viscous energy needed to separate the material in droplets is finite, i.e. it is larger than a finite value whatever the rate of the transformation, which obviously can preclude the development of the instability if surface tension effects are not sufficiently large. In order to appreciate the tendency of the fluid to separate a jet of YSF (initially a cylinder of radius $R$ ) in droplets we can rely on rough estimates: the surface energy created per droplet is of the order of $2 \sigma \pi R^{2}$ (where $\sigma$ is the surface tension of the fluid, see Appendix 1); the viscous energy needed to deform the fluid during this separation is of the order of that needed to deform the volume of a strain 1 , which would be $\tau_{c} \Omega$ (with $\Omega$ the droplet volume, which is of the order of $4 \pi R^{3} / 3$ ). We deduce that YSF droplets should spontaneously form if $\tau_{c}<<\sigma / R$. This means that a jet of a few millimeter diameter will easily separate in droplets if the yield stress is smaller than a few Pascals. Otherwise the jet of YSF will tend to keep its cylindrical shape, possibly deformed when reaching a solid surface and then forming a coil (see Rahmani et al 2011).

However, in most cases, gravity will start to play a role beyond some critical length of the jet, and will lead to the rapid separation in droplets. This effect can easily be understood. According to the standard 3D expression for the constitutive equation (see Section 2 ) the critical normal stress needed to induce an elongational flow of a cylinder of YSF is equal to $\sqrt{3} \tau_{c}$. On the other hand the normal stress acting on a layer of fluid in a jet is equal to the weight of fluid situated below this layer, divided by the jet section area, i.e. $\rho g l$. When this normal stress due to fluid weight is larger than $\sqrt{3} \tau_{c}$, an elongational flow starts, which tends to decrease the section area, so that the difference between the two stresses increases, leading to faster elongation. Since we are dealing with strongly shearthinning fluids, this leads to a catastrophic decrease of the fluid section area and finally a separation of the material in that region in a very short time (see Figure 7).

A similar effect occurs when one attempts to elongate a yield stress fluid volume initially comprised between two parallel plates that are then moved progressively away from each other. Instead of simple uniaxial elongational flow we observe a separation of the material into two approximately conical parts attached to the plates: see Figure 8. 
In fact this experiment provides a deeper insight in the process of separation of a volume of YSF into two parts: we can see that the whole material is initially deformed but soon the regions close to the disk surfaces and situated at the periphery stop flowing, since the shape of these regions remain fixed during the next steps; a similar process goes on for new fluid volumes (now closer to the central axis) as the disks are moved farther, so that increasing volumes stop flowing along the disks, and in the final step there remains only a small central region which is deformed and eventually breaks (see Figure 8). Thus this flow may be seen in a different way: it can be considered as the penetration, through the YSF, of a radial air finger towards the central axis. This necking process is reminiscent of the ductile breakage observed for example with metals during a traction test (Nadai 1950). We find again a strong analogy of the properties of slow YSF flows with those of plastic materials.

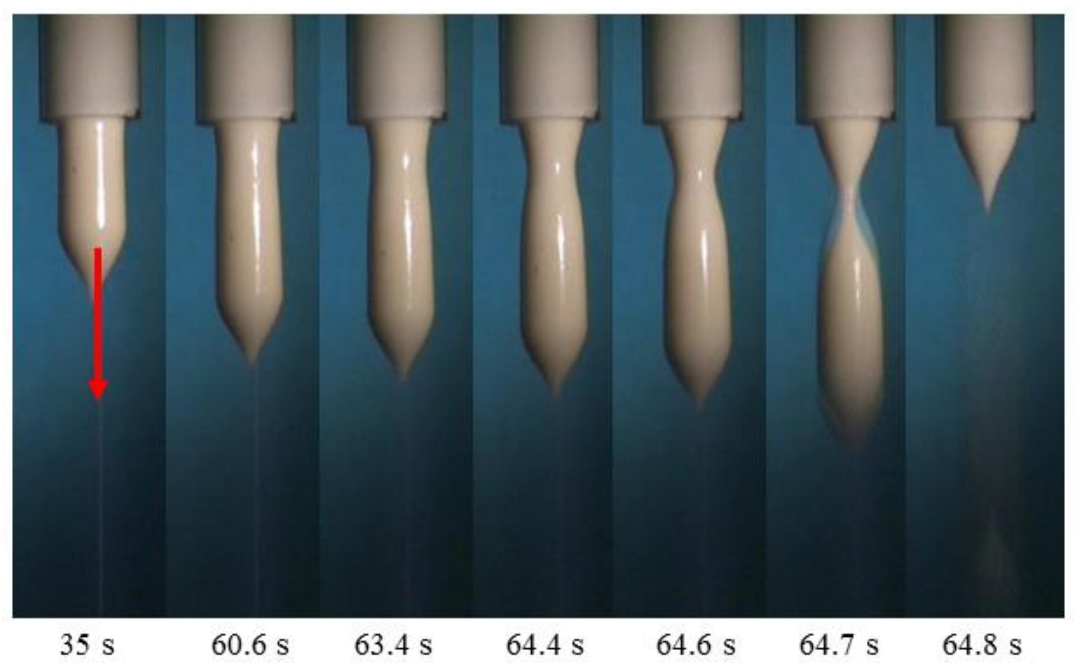

Figure 7: Successive views of a mayonnaise sample at different times during extrusion. The initial time $(0 \mathrm{~s})$ corresponds to the separation of the previous extrudate. [Figure 1 of Coussot and Gaulard 2005].

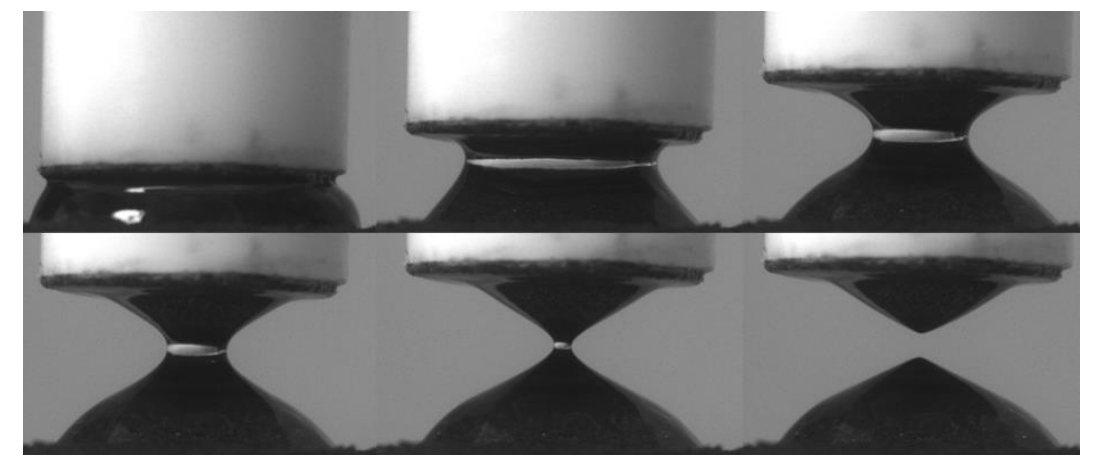

Figure 8: Successive views of a YSF shape during a traction test. [Courtesy Jalila Boujlel]

\section{Saffman-Taylor instability}

The Saffman-Taylor instability (STI) is observed when a fluid pushes a more viscous fluid in a confined geometry. The term confined here means that the distance between the solid walls is much smaller than the characteristic length in the flow direction. Such boundary conditions are typically 
encountered in porous media or between two parallel plates (i.e. Hele-Shaw cell). Under stable conditions the length of the interface between the two fluids remains minimal, so that it is straight for a flow in a single direction, or circular for a radial flow. When the STI develops, the interface evolves in the form of fingers which, will then evolve in different ways depending on the rheological behavior of the material (Lindner et al. 2002).

The most frequent situation for which the STI is observed with a YSF is the separation of two plates initially in contact with a thin layer of paste; as the plates are moved away the layer thickness increases, which induces a radial flow towards some central position; if the distance between the plates is sufficiently small, the radial velocity is much larger than the axial one, so that the flow approximately corresponds to a radial flow driven by the air entering the gap, which corresponds to the boundary conditions under which the STI can be considered. This phenomenon can be observed as soon as some thin layer of paint, glue, puree, yoghurt, is squeezed between two solid surfaces (a tool, a spoon, etc) which are then separated: we get a characteristic fingering shape as shown in Figure 9. Note that it is possible to observe such pictures because the fluid leaves arrested regions behind the flow front, which finally give this definitive shape. This contrasts with simple liquids for which the fingers soon relax under the action of wetting effects and a uniform layer rapidly reforms.

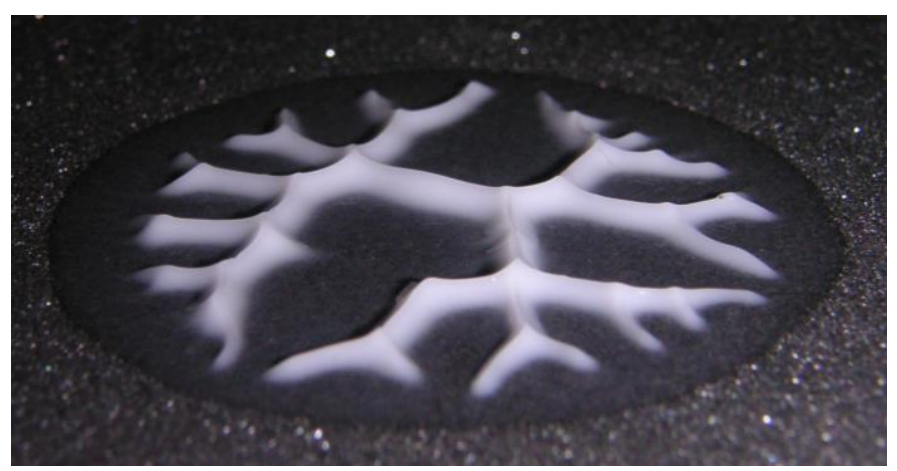

Figure 9: Fingering aspect of the remaining deposit of yield stress fluid after separation of two plates initially squeezing a thin fluid layer. [Courtesy Quentin Barral]

For simply viscous fluids the origin of the instability is that any unevenness (local curvatures) of the interface allows a subsequent easier advance of the fluids in front of the curvature in the flow direction, while for a curvature in the opposite direction the motion is more difficult, it requires more pressure because the volume of the more viscous fluid to push is larger. It results that the perturbation will tend to develop further, except if surface tension, which on the contrary works against the deformation of the initial interface, is sufficient to counterbalance this effect. We can express this mathematically following the approach of Homsy (1987). We consider a material pushed by an inviscid fluid and flowing in a given direction $x$ between two parallel plates separated by a small distance $2 b$. We assume that the interface (initially situated in $x=0$ ) undergoes a small perturbation $\varepsilon=\varepsilon_{0} \exp (i k z)$ in which $k$ and $\varepsilon_{0}$ are two parameters. $2 \pi / k=\lambda$ is the wavelength of the perturbation. This induces a curvature of the interface approximately equal to $1 / \varepsilon^{\prime \prime}=-1 / k^{2} \varepsilon$, which leads to an additional pressure difference along the interface $-\sigma k^{2} \varepsilon$. Let us consider a small "band" of fluid along the direction $x$. The additional pressure specifically needed to displace this band of the additional local distance $\varepsilon$ is found from a momentum balance on the small volume displaced: $2 b d p=2 \tau_{w} \eta$, in which $\tau_{w}$ is the shear stress along the wall. Finally the total additional 
pressure required is $d p=\left[\left(\tau_{w} / b\right)-\sigma k^{2}\right] \varepsilon_{0}$ at the front. If this term is of the sign of $\varepsilon$, i.e. if $\lambda>\lambda c$ with $\lambda_{c}=2 \pi \sqrt{b \sigma / \tau_{w}}$, the additional pressure will tend to push even more the regions which have already been curved towards positive values of $x$, the perturbation will be amplified and the instability will develop. We see that for the instability to develop, the viscous forces must be sufficiently large compared to the forces resulting from surface tension. For a simple fluid this means that the instability will anyway develop beyond some critical velocity.

It is tempting to use such an approach for a yield stress fluid, remarking that as soon as the material is flowing $\tau_{w}$ is larger than $\tau_{c}$, which implies that the instability for a YSF would appear at any flow rate if $\tau_{c}>\sigma b(2 \pi / L)^{2}$. More complete and precise theoretical or numerical approaches of this instability for yield stress fluids have been developed, still within a fluid mechanics frame, i.e. essentially taking into account the behavior of the material in its liquid regime. This led to detailed instability criteria and descriptions and analysis of various trends (Coussot 1999, Fontana et al 2013, Ebrahimi et al. 2016). The validation of these conclusions by confrontation with experimental data remains extremely limited, and when this was done a reasonable agreement for the finger size (Lindner et al. 2000, Derks et al 2003, Maleki-Jirsaraei et al 2005), but a strong discrepancy was found concerning the instability criterion (Barral et al 2010), suggesting some important effect is missed.

Thus, for a YSF we have an original situation as we can get a "hydrodynamic" instability at vanishing velocity. Another original aspect of this instability for YSF is that at sufficiently low flow rate the fingering process leaves arrested fluid volumes behind the advancing front. This again results from the specific solid-liquid behavior of YSF: as soon as some fingering starts, the regions for which the curvature is in the direction opposite to flow, require a larger pressure gradient to flow (see above equation for additional pressure); if the (average) flow rate is imposed at a moderate value the pressure to maintain the flow in the other regions will be smaller than the critical pressure to induce motion in the above regions (situated behind). This difference increases as the fingers grow so that the regions behind will remain definitively arrested.

Actually, these observations suggest that the description, within the frame of fluid mechanics, of this instability for YSF, might not be appropriate. More precisely we have important differences with the assumptions at the basis of the standard fluid mechanics approach. The instability develops from an initial state at rest, which means that the material is in its solid regime when one looks at the possible destabilization; a linear stability analysis in fluid mechanics neglects the initial deformation required to reach the liquid regime, whereas it relies on the analysis of the evolution of a small perturbation of the interface, which is associated with the very first deformation of the material, still likely in its solid regime in most of its volume. Obviously, in this context, the standard fluid mechanics approach assumes that the evolution of this perturbation takes place while all the material is flowing (possibly at different velocities), and thus does not take into account the arrested regions.

Finally, taking into account these features, we can propose a different view of the problem, that seems closer to the involved physical processes. Since the material regions along the air fingers remain at rest all along the advance of the rest of the material, we may see the process as the progression of air fingers penetrating the YSF initially at rest. Under such conditions we expect that, as for an object penetrating a bath of YSF (see Section 2) the material will be in its liquid regime only in a small volume along the finger. But the situation is likely even more critical here as this is not a solid object which penetrates the material: since air induces a negligible stress along the finger sides, we expect a liquid regime to exist at a given time only in the newly deformed region around the finger tip. This would imply that the treatment of this problem has nothing to do with the usual treatment in fluid mechanics, it is essentially a matter of flow localization in a large solid volume 
(note that for a traction test this assumes that the aspect ratio is very small, so that residual elongational flow does not allow the material to reach its liquid regime).

\section{Rayleigh-Taylor instability (RTI)}

The Rayleigh-Taylor Instability (RTI) is an instability which occurs when a denser fluid rests on top of a lighter one (Rayleigh 1883, Sharp 1984) as a result of gravity effects. As it develops, the two fluids penetrate one another, in the form of fingers. The instability is driven by density difference and the acceleration to which the fluids are submitted, while surface tension provides a stabilizing effect. A example of situation where this instability can be encountered is in oil well cementing operations, in which yield stress fluids of different densities (drilling muds and cement, e.g), which behave as solids at rest, may be pumped into the well in an ill-favored density order (Bittleston and Guillot 1991).

For Newtonian fluids the instability is well described. The interface starts to deform and give rise to fingers penetrating the initial volume of the other phase. The wavelength of these fingers decreases when the density difference increases or interfacial tension decreases. The fluid viscosities do not play any role in the instability criterion, but have an impact on the dynamics. It is worth noting that stable situations may be obtained in some cases when the above fluid has a density larger than the bottom fluid, if the size of the interface is sufficiently small. This explains why the liquid may not flow from a suddenly turned over bottle, if its neck is sufficiently small. A typical example of the development of the instability for two simple liquids, just beyond the stable regime, is shown in Figure 10a: a regular sinusoidal interface forms with a wavelength slightly larger than the bath length, and an amplitude growing in time. Beyond some amplitude the fingers tend to accelerate and finally form jets rapidly penetrating the other phase, with mushroom-like shape after some distance.

For a yield stress fluid the instability characteristics differ significantly from those above described. Let us consider a simple (denser) liquid placed above a bath of a YSF. Under some conditions the interface does not move at all, even after a long time. This corresponds to a perfectly stable situation. Under conditions that can be slightly different (in particular concerning the density difference, the yield stress) the interface destabilizes. A very fast motion of the YSF into the Newtonian solution occurs in the form of mushroom-like bursts (see Figure 10b). These bursts penetrate the liquid and reach the top of the tank in a time of the order of one second. At the same time (although not visible in these images) more or less symmetric liquid fingers penetrate the YSF bath. 


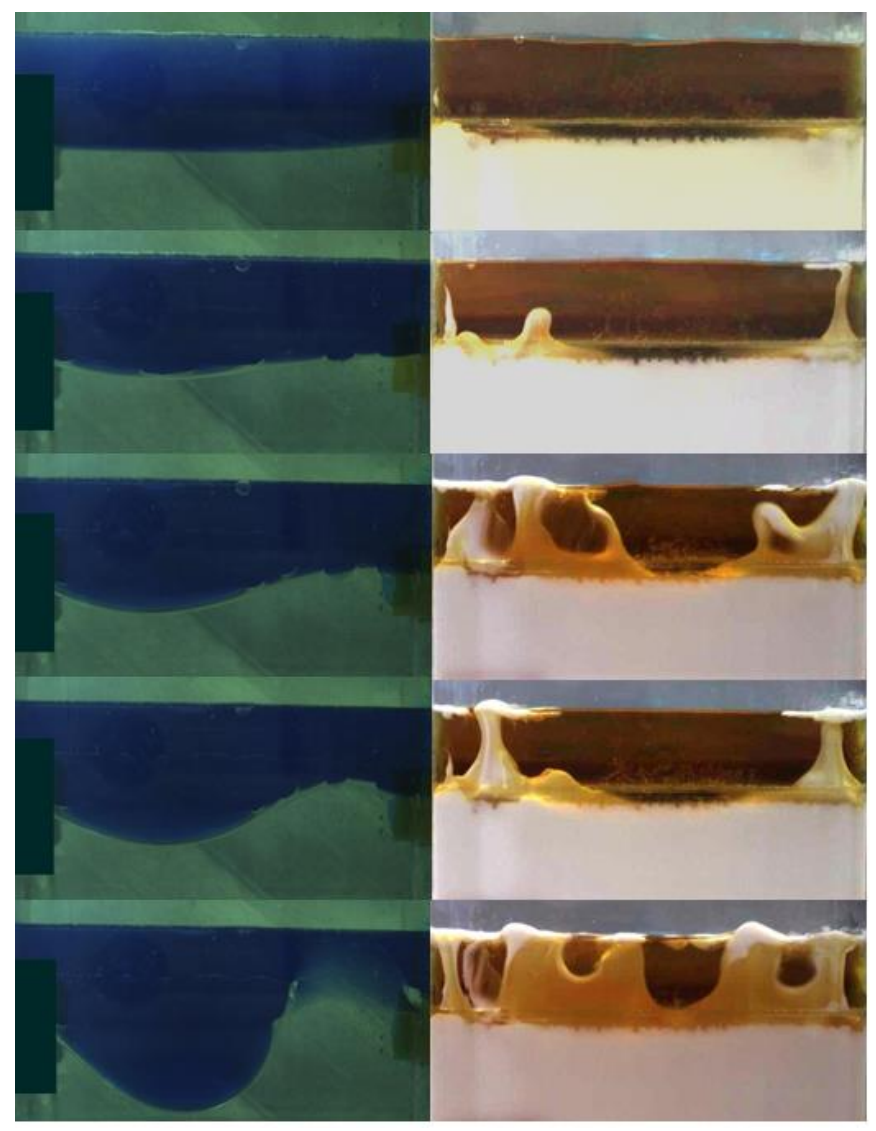

Figure 10: RTI development for (a, left) viscous Newtonian fluid (Silicone oil, 0.35 Pa.s viscosity) initially below a denser colored water-ethanol solution $(\Delta \rho / \rho=1.3 \%)$ at times (from top to bottom): 0 (mid-time of gate opening), $6,12,18$, and $24 \mathrm{~s}$; and (b, right) a concentrated (white) emulsion ( $\tau_{c}=9.6 \mathrm{~Pa}$ ) initially below a (brown) denser salt solution $\left(\Delta \rho=600 \mathrm{~kg} \cdot \mathrm{m}^{-3}\right)$ at times: $0,1.9,3,3.8$, and 4.5s. Figure 1 of Maimouni et al 2016.

Some features are remarkably different from those usually observed with simple liquids: i) even just beyond the stable conditions the instability develops in the form of a catastrophic process as described above; ii) in unstable cases, the size of the unstable bursts (i.e. half the instability wavelength) approximately keeps a constant value, significantly smaller than the interface length, whatever the experimental conditions (tank size, density difference, yield stress).

For simple fluids the linear stability analysis of the problem consists to assume some small perturbation of the interface and see how it should evolve. This leads to a criterion for the instability to occur which corresponds to a comparison between capillary effects and gravity effects. Obviously such a criterion cannot work for a YSF, since the yield stress, like capillary effects, tends to resist to the interface deformation, which is naturally associated with bulk deformation. In fact, once again, this is not exactly the yield stress, but the strength of the material in its solid regime, which acts to damp a possible perturbation of the interface from its initial solid state.

Actually, this instability has already been studied for solids, because it relates to various observations of natural phenomena in which a liquid apparently opens its way through a layer of denser solid material above it: slowly accreting neutron stars (Blaes et al 1990), volcanic island formation (Marsh 1979), salt dome formation (Zaleski and Julien 1992), and more generally magmatic diapirism in the Earth's mantle and continental crust (Piriz et al. 2013, Burov and Molnar 2008). In this frame, assuming negligible surface tension effects, it was remarked that two main origins of destabilization can be considered depending on the material behavior. 
If the solid is essentially elastic we can expect an instability to occur when the gravity effects (proportional to the density difference and the amplitude of the perturbation) are larger than the elastic force associated with the material deformation, which gives: $\Delta \rho g>4 \alpha \pi G / L$, with here $\alpha$ between 1 and 2 depending on boundary conditions (Terrones 2005, Piriz et al 2005, Robinson and Swegle 1989). Using soft elastic solids, the overall validity of this approach was proved experimentally but the wavelength was shown to be smaller than expected from theory, and depending on uncontrollable, slight disturbances of the surface (Mora et al 2014).

If the solid is plastic we can expect an instability to develop as soon as the critical deformation $\left(\varepsilon_{0}\right)$ associated with the elastic limit has been overcome. This leads to the following criterion of instability: $g \Delta \rho>\beta \tau_{c} / \varepsilon_{0}$, with $\beta$ in the range $0.5-2$ depending on the sample aspect ratio (Piriz et al 2005, Robinson and Swegle 1989, Dimonte et al 1998). Finally we can conclude from these approaches that for a simple solid at least one of the two above criteria might need to be fulfilled for the instability to develop, but the experimental validation of this conclusion is still extremely limited.

Such an approach should be applicable for YSF, which are also elastoplastic solid below the yield stress. However it was recently shown (Maimouni et al 2016) that for YSF a specific phenomenon occurs, which results from their particular rheological behavior: the force needed to induce the initial penetration of an object in a YSF bath is much smaller than that expected for the deformation of the same volume of an elastic material with the same elastic modulus. It was then suggested that, in consistency with the observations for larger penetration depth (Boujlel et al 2012), as soon as a perturbation (say, a liquid finger), tends to penetrate the YSF, this induces locally, i.e. in a thin layer of YSF around the finger, a solid-liquid transition, due to the large deformations undergone by the material in that region. Further penetration of the finger does not need to deform widely the YSF bath, a moderate deformation along with this flow localization can ensure the finger development, and the required force is smaller than for a pure elastic material. Moreover, since the force needed for this penetration finds its essential origin in the progression of the finger tip, this force remains almost constant, whereas the driving (gravity) force due to density difference between the two materials increases proportionally to the finger length. This explains the catastrophic phenomenon observed in practice (see above and Figure 10b): as soon as they have been formed the fingers very rapidly develop.

\section{Conclusion}

We have seen that YSF develop original flow features (as compared to simple fluids) under various boundary conditions. This analysis made it possible to highlight some of their common original characteristics: i) a transition from a solid regime to a flowing regime which does not correspond to a true "liquid state", the flow in this regime may rather be seen as a succession of solid states during very large deformation; ii) a strong tendency to localization of the yielded regions in some small region of the material while the rest of the material undergoes some deformation in its solid state; iii) the deformation of YSF interface with another fluid, in the form of fingers tending to penetrate the material via a local liquefaction process. Moreover the flow characteristics (rescaled by the macroscopic velocity) often appear almost independent of macroscopic velocity in the slow flow range.

Finally these observations suggest that slow flows of YSF are a kind of extension of plastic flows for very large deformations and without irreversible changes of the structure. This suggests that the field of plasticity and the field of slow flows of YSF could benefit from each other. For example, for some flow problems it seems likely that analytical approaches for predicting the flow characteristics of YSF could be developed on the basis of tools used in the field of plasticity, such as the so-called slip line 
theory (Lubliner 1990). Conversely these observations suggest that our knowledge of YSF flows could benefit to the description of plastic flows or to the validation of plastic flow modelling.

\section{Appendix 1. Interfacial tension of a yield stress fluid with another phase}

Since interfacial tension is related to the interactions between the molecules situated along the interface (Israelachvili 2001) and since YSF are made of suspended elements in a liquid, it is natural to consider that the interfacial tension of a YSF with another (simple) phase is given by that of the interstitial liquid with this other phase, since there is always at least a few layers of liquid molecules surrounding the suspended elements. This assumption appears to be in agreement with existing measurements (Yoshitake et al 2008, Jalila and Coussot 2013, Aytouna et al 2013, Jorgensen et al 2015). It remains that a direct measure of interfacial tension with YSF is a challenge because, in such materials, there might exist, even at rest, residual stresses which can preclude a straightforward appreciation of interfacial stresses.

\section{References}

Aytouna M, Paredes J, Shahidzadeh-Bonn N, Moulinet S, Wagner C., Amarouchene Y, Eggers J, Bonn D (2013) Drop formation in non-Newtonian fluids, Phys. Rev. Lett., 110:034501

Balmforth N, Frigaard I, Ovarlez G (2014), Yielding to stress: Recent developments in viscoplastic fluid mechanics, Annual Review of Fluid Mechanics, 46:121-146

Barral Q, Boujlel J, Chateau X, Rabideau BD, Coussot P (2010) Adhesion of yield stress fluids, Soft Matter 6:1343-1351

Benbow J, Bridgewater J (1993) Paste flow and extrusion, Clarendon Press, Oxford

Bittleston S, Guillot D (1991) Mud Removal: Research Improves Traditional Cementing Guidelines, Oilfield Review, 3:44-54

Blaes O, Blandford R, Madau P, Koonin S (1990) Slowly accreting neutron-stars and the origin of gamma-ray bursts, Astrophys. J., 363, 612-627

Boger DV, and Walters K (1993) Rheological phenomena in focus, Elsevier, Amsterdam

Bonn D, Paredes J, Denn M, Berthier L, Divoux T, Manneville S (2017) Yield Stress Materials in Soft Condensed Matter, Rev. Modern Phys., 89, 035005

Boujlel J, Coussot P (2013) Measuring the surface tension of yield stress fluids, Soft Matter, 9:58985908

Boujlel J, Maillard M, Lindner A, Ovarlez G, Chateau X, Coussot P (2012), Boundary layer in pastes Displacement of a long object through a yield stress fluid, J. Rheol., 56:1083-1108

Burov EB, Molnar P (2008) Small and large-amplitude gravitational instability of an elastically compressible viscoelastic Maxwell solid overlying an inviscid incompressible fluid: Dependence of growth rates on wave number and elastic constants at low Deborah numbers, Earth Planetary Sci. Lett., 275,370

Chevalier T, Rodts S, Chateau X, Boujlel J, Maillard M, Coussot P (2013), Boundary layer (shear-band) in frustrated viscoplastic flows, EPL, 102:48002

Cloitre M, Bonnecaze RT (2017) A review on wall slip in high solid dispersions, Rheol. Acta, 56:283305

Cottrell AH (1964) The mechanical properties of matter, Wiley, New York 
Coussot P (1999) Saffman-Taylor instability for yield stress fluids, Journal of Fluid Mechanics, 380:363-376

Coussot P (2014) Yield stress fluid flows: a review of experimental data, J. Non-Newt. Fluid Mech., 221:31-49

Coussot P (2017) Bingham's heritage, Rheol. Acta, 56:163-176

Coussot P, and Gaulard F (2005) Gravity flow instability of viscoplastic materials: the "ketchup drip", Physical Review E, 72:031409

Coussot P, Tabuteau H, Chateau X, Tocquer L, and Ovarlez G (2006) Aging and solid or liquid behavior in pastes, J. Rheol., 50:975-994

Coussot P, and Ovarlez G (2010) Physical origin of shear-banding of jammed systems, European Physical Journal E, 33:183-188

Coussot P, Tocquer L, Lanos C, Ovarlez G (2009) Macroscopic vs local rheology of yield stress fluids, Journal of Non-Newtonian Fluid Mechanics, 158:85-90

Derks D, Lindner A, Creton C, Bonn D (2003) Cohesive failure of thin layers of soft model adhesives under tension, J. Appl. Phys., 93:1557-1566

Dimonte G, Gore R, Schneider M (1998) Rayleigh-Taylor instability in elastic-plastic materials, Phys. Rev. Lett., 80:1212-1215

Ebrahimi B, Mostaghimi P, Gholamian H, Sadeghy K (2016) Viscous fingering in yield stress fluids: a numerical study, J. Eng. Math., 97:161-176

Fontana JV, Lira SA, Miranda JA (2013) Radial viscous fingering in yield stress fluids: onset of pattern formation, Phys. Rev. E 87:013016

Hébraud P, Lequeux F, Munch JP, Pine DJ (1997) Yielding and rearrangements in disordered emulsions, Phys. Rev. Lett. 78:4657-4660

Homsy GM (1987) Viscous fingering in porous media, Ann. Rev. Fluid Mech., 19:271-311

Israelachvili JN (2001) Intermolecular and surface forces, Academic Press, Amsterdam

Jorgensen L, Le Merrer M, Delanoe-Ayari H, Barentin C (2015) Yield stress and elasticity influence on surface tension measurements, Soft Matter, 11:5111-5121

Lidon P, Villa L, Manneville S (2017) Power-law creep and residual stresses in a carbopol gel, Rheol. Acta, 56:307-323

Lubliner J (1990) Plasticity theory, Macmillan, New York

Lindner A, Bonn D, and Coussot P (2000) Viscous fingering in a yield stress fluid, Physical Review Letters, 85:314-317

Lindner A, Bonn D, Poire EC, Ben Amar M, Meunier J (2002) Viscous fingering in non-Newtonian fluids, 469:237-256

Liu AJ, Nagel SR (1998) Jamming is not just cool any more, Nature, 396:21-22

Maillard M (2015) Spreading flows of yield stress fluids, PhD thesis, Univ. Paris-Est (in French)

Maillard M, Mézière $C$, Moucheront $P$, Courrier C, Coussot $P$ (2016) Blade-coating of yield stress fluids, J. Non-Newt. Fluid Mech., 237:16-25

Maimouni I, Goyon J, Lac E, Pringuey T, Boujlel J, Chateau X, Coussot P (2016) Rayleigh-Taylor instability in elastoplastic solids: a local, catastrophic process, Phys. Rev. Lett., 116:154502

Maloney CE, Lemaître A (2006) Amorphous systems in athermal, quasistatic shear, Phys. Rev. E, 74:016118

Maleki-Jirsaraei N, Lindner A, Rouhani S, Bonn D (2005) Saffman-Taylor instability in yield stress fluids, J. Phys. Cond. Matt., 17:S1219-S1228 
Marsh BD (1979) Island-arc development - Some observations, experiments, and speculations, J. Geol., 87:687-713

Moller P, Fall A, Chikkadi V, Derks D, Bonn D (2009), An attempt to categorize yield stress fluid behavior, Philosophical Trans. Royal Soci. A- Mathematical Physical and Engineering Sciences, 367:5139-5155

Mora S, Phou T, Fromental JM, Pomeau Y (2014) Gravity Driven Instability in Elastic Solid Layers, Phys. Rev. Lett., 113:178301

Nadai A (1950) Theory of flow and fracture of solids, McGraw Hill, New York

Oldroyd JG (1947) A rational formulation of the equations of plastic flow for a Bingham solid, Proc. Cambridge Philosophical Society, 43:100-105

Ovarlez G, Cohen-Addad S, Krishan K, Goyon J, Coussot P (2013) On the existence of a simple yield stress fluid behavior, J. Non-Newt. Fluid Mech., 193:68-79

Ovarlez G, Rodts S, Chateau X, Coussot P (2009) Phenomenology and physical origin of shearlocalization and shear-banding in complex fluids, Rheologica Acta, 48:831-844

Piriz AR, López Cela JJ, Cortázar OD, Tahir NA, Hoffmann DHH (2005) Rayleigh-Taylor instability in elastic solids, Phys. Rev. E, 72, 056313

Piriz AR, Sun YB, Tahir NA (2013) Rayleigh-Taylor stability boundary at solid-liquid interfaces, Phys. Rev. E, 88:023026

Rahmani Y, Habibi M, Javadi A, Bonn D (2011) Coiling of yield stress fluids, Phys. Rev. E 83:056327

Rayleigh SJW (1883) Investigation of the character of the equilibrium of an incompressible heavy fluid of variable density, Proc. London Math. Soc., 14:170-177

Robinson AC, and Swegle JW (1989) Acceleration instability in elastic-plastic solids 2. Analytical techniques, J. Appl. Phys. 66:2859-2872

Sharp DH (1984) An overview of Rayleigh-Taylor instability, Physica, 12D:3-18

Sollich P, Lequeux F, Hébraud P, Cates ME (1997) Rheology of Soft Glassy Materials, Phys. Rev. Lett., 78,2020

Tabor D (1991) Gases, liquids and solids (Cambridge, Cambridge University Press)

Terrones G (2005) Fastest growing linear Rayleigh-Taylor modes at solid/fluid and solid/solid interfaces, Phys. Rev. E, 71:036306

Yoshitake Y, Mitani S, Salai K, Takagi K (2008), Surface tension and elasticity of gel studied with laserinduced surface-deformation spectroscopy, Phys. Rev. E, 78:041405

Zaleski S, Julien P (1992) Numerical simulation of Rayleigh-Taylor instability for single and multiple salt diapirs, Tectonophysics, 206:55-69

Zhang X, Lorenceau E, Basset P, Bourouina T, Rouyer F, Goyon J, Coussot P (2017) Wall slip of softjammed systems: a generic, apparent simple shear process, to appear in Phys. Rev. Lett. 